\title{
Changes in circulating microRNA-126 during treatment with chemotherapy and bevacizumab predicts treatment response in patients with metastatic colorectal cancer
}

T F Hansen ${ }^{* 1,2}$, A L Carlsen ${ }^{3}, \mathrm{~N} \mathrm{H} \mathrm{H} \mathrm{Heegaard}{ }^{3}$, F B Sørensen ${ }^{2,4}$ and A Jakobsen ${ }^{1,2}$

${ }^{1}$ Department of Oncology, Vejle Hospital, Part of Lillebaelt Hospital, Vejle, Denmark; ${ }^{2}$ Institute of Regional Health Research, University of Southern Denmark, Denmark; ${ }^{3}$ Department of Clinical Biochemistry, Immunology \& Genetics, Statens Serum Institute, Copenhagen, Denmark and ${ }^{4}$ Department of Clinical Pathology, Vejle Hospital, Part of Lillebaelt Hospital, Vejle, Denmark

Background: This study investigated the predictive value of circulating microRNA-126 (cir-miRNA-126) in patients with metastatic colorectal cancer $(\mathrm{mCRC})$ treated with first-line chemotherapy combined with bevacizumab.

Methods: The study included 68 patients. Blood samples (plasma) were collected before the treatment initiation, at the first clinical evaluation after 3 weeks and at progression. Levels of cir-miRNA-126 were determined by qRT-PCR after purification of total RNA from plasma. Primary clinical end points were response rates evaluated according to the Response Evaluation Criteria In Solid Tumours (RECIST) and progression-free survival (PFS).

Results: Changes in circulating miRNA-126 during treatment were predictive of tumour response. Non-responding patients had a median increase in cir-miRNA-126 of 0.244 (95\% confidence interval $(\mathrm{Cl}), 0.050-0.565$ ) compared with a median decrease of $-0.374(95 \% \mathrm{Cl},-0.472$ to -0.111$)$ in the responding patients, $P=0.002$. A significant positive correlation was demonstrated by comparing the changes in tumour size with the changes in cir-miRNA-126, $r=0.48, P=0.0001$. Grouping the patients according to the changes in cir-miRNA-126 disclosed a borderline significant separation of the groups in the PFS analysis favouring patients with decreasing miRNA-126 levels, hazard ratio (HR) $0.60(95 \% \mathrm{Cl}, 0.33-1.09), P=0.07$.

Conclusions: The present results indicate that changes in cir-miRNA-126 during treatment are related to the response to chemotherapy and bevacizumab in patients with $\mathrm{mCRC}$, thus representing a possible biomarker for the resistance to anti-angiogenic containing treatments.

Chemotherapy combined with bevacizumab is a common choice for patients with metastatic colorectal cancer (mCRC). Unfortunately, the benefit is rather limited and it has so far not been possible to identify patients likely to benefit from this regimen. Candidate biomarkers should reflect the anti-angiogenic effect of therapies, preferentially at an early stage during treatment, and be obtainable through minimal invasive procedures.

MicroRNAs are short non-coding single-stranded RNAs that regulate protein translation (Esquela-Kerscher and Slack, 2006;
Filipowicz et al, 2008; Kong et al, 2012). Though exerting their functions in the intracellular compartment, miRNAs have also been identified in several extracellular compartments (Chen et al, 2008; Mitchell et al, 2008; Weber et al, 2010). Circulating extracellular miRNAs are stable in body fluids presumably due to their incorporation in exosomes/microvesicles or protein complexes, protecting them from degradation by endogenous RNase activity (Mitchell et al, 2008; Kosaka et al, 2010). Evidence suggests that these small vesicles act as mediators of intercellular 
communications (Cocucci et al, 2009) through the transfer of mRNA and miRNAs from the vesicles to the recipient cells (Valadi et al, 2007). Circulating miRNAs are also detectable in patients with colorectal cancer (CRC; Chen et al, 2008). The clinical importance of circulating miRNAs as biomarkers of CRC ( $\mathrm{Ng}$ et al, 2009), and pointers as to presence or absence of metastatic disease (Wang and $\mathrm{Gu}, 2012$ ), are currently under investigation. The role of circulating miRNAs as predictive biomarkers in patients with CRC in general, and of the effect of anti-angiogenic therapies specifically, is largely unknown.

MicroRNA-126 has a high endothelial cell (EC) specificity (Wienholds et al, 2005; Zhu et al, 2011). It is involved in EC proliferation, migration, survival, and in the regulation of blood vessel integrity (Fish et al, 2008; Wang et al, 2008). Shortly after demonstrating the content of miRNAs in small vesicles released from human cells (Valadi et al, 2007), miRNA-126 was also shown to be contained in the plasma exosomes/microvesicles in healthy individuals, and a fraction of these originated from ECs (Hunter et al, 2008). Circulating miRNA-126 (cir-miRNA-126) in microvesicles/exosomes is able to induce angiogenesis in neighbouring ECs using a paracrine mechanism by fusing with the cell membrane of the target ECs (Mocharla et al, 2013).

Bevacizumab is an antibody involved in targeting the vascular endothelial growth factor A (VEGF-A). It prevents the binding of VEGF-A to the receptor (VEGFR-2), which is a main mediator of angiogenic signalling (Ferrara et al, 2003). The signalling cascade initiated by the binding of VEGF-A to VEGFR-2, is regulated by miRNA-126 (Fish et al, 2008; Wang et al, 2008). Microvesicles are released from healthy as well as damaged cells, and elevated levels of EC-derived microvesicles have been correlated with vascular damage (Bakouboula et al, 2008). Considering this and the fact that miRNA126 is highly specific for ECs, it is likely that cir-miRNA-126 reflects EC turnover. In patients with $\mathrm{mCRC}$ treated with anti-angiogenic drugs, changes in cir-miRNA-126 during treatment may therefore serve as a biomarker of bevacizumab (anti-VEGF-A) efficacy.

The aim of the current study was to analyse the predictive value of cir-miRNA-126 during first-line chemotherapy and bevacizumab in patients with mCRC.

\section{MATERIALS AND METHODS}

Reporting in this study is in accordance with the REMARK (McShane et al, 2005) and BRISQUE (Moore et al, 2011) criteria.

Patients and study treatment. This study consecutively included untreated patients with histologically verified mCRC planned for first-line chemotherapy (XELOX) combined with bevacizumab, with an overall aim of identifying predictive biomarkers. All patients were screened for eligibility and inclusion was possible for 68 (Figure 1) in the inclusion phase from March 2010 through March 2013. All patients were $>18$ years and presented with normal hepatic, renal, and bone marrow function. Previous malignancies diagnosed within 5 years (except non-melanoma skin cancer and carcinoma in situ of the cervical uterus) were the exclusion criteria.

Chemotherapy consisted of capecitabine $1000 \mathrm{mg} \mathrm{m}^{-2}$ twice daily on days 1 through 14 (28 doses) of a 21-day cycle and oxaliplatin $130 \mathrm{mg} \mathrm{m}^{-2}$ as a 2-hour intravenous infusion on day 1 . Bevacizumab was given as an infusion $\left(7.5 \mathrm{mg} \mathrm{kg}^{-1}\right)$ on day 1 of each treatment cycle. Treatment was continued until disease progression or unacceptable toxicity, but treatment-free intervals were accepted after six cycles, if requested by the patient.

All data were recorded according to good clinical practice. The study was approved by the Regional Scientific Ethical Committee and the Danish Data Protection Agency (S-20100005), and informed consent was obtained from all patients enrolled in the study.

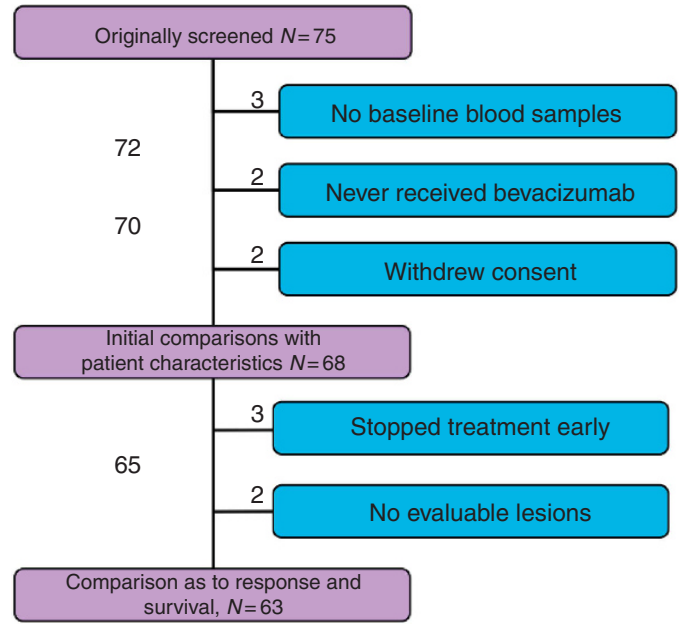

Figure 1. Consort diagram of the study population.

Evaluation of treatment efficacy. Response rates (RR) were assessed according to the Response Evaluation Criteria In Solid Tumours (RECIST 1.1; Eisenhauer et al, 2009). Evaluations were based on clinical and radiologic examination using CT scans of the chest and abdomen. Responding patients were classified as having complete response (CR) or partial response (PR), whereas nonresponding patients were classified as stable disease (SD) or progressive disease (PD).

Blood samples. Sampling of peripheral blood was carried out before treatment (baseline) and at each clinical evaluation (after every 3 weeks) until progression. To standardise the comparability, three time points (baseline, first clinical evaluation after 3 weeks, and progression) were chosen for the present study. Blood samples were available for analysis at baseline for all 68 patients (100\%), at first clinical evaluation in 63 patients (93\%), and at progression in 47 patients $(69 \%)$.

Venous blood was drawn from the antecubital area and collected in $6 \mathrm{ml}$ EDTA-containing tubes, spun down for $10 \mathrm{~min}$. at $2500 \mathrm{~g}$, and plasma was transferred to Greiner tubes (SigmaAldrich, St Louis, MO, USA) and frozen immediately at $-80^{\circ} \mathrm{C}$. The median storage time from sampling to analysis was 2.1 years. Samples were transported on ice from storage for analysis.

miRNA-126 analyses. Analyses of miRNA-126 were performed by staff who were unaware of the patient outcome.

Total RNA purification kit (Norgen Biotek Corp., Thorold, ON, Canada) was used to purify RNA from $100 \mu$ l of each plasma sample according to the manufacturer's instructions with small modifications: $10 \mathrm{~mm}$ dithiothreitol (Sigma-Aldrich Co. LLC, Steinheim, Germany) and $1.7 \mathrm{pM}$ synthetic C. elegans miR-54 and -238 (TAG Copenhagen A/S, Denmark) were added into the lysis buffer. RNAse inhibitor $(1 \mu \mathrm{l})\left(20 \mathrm{U} \mu \mathrm{l}^{-1}\right.$, Applied Biosystems, Foster city, CA, USA) was added to every elution tube before eluting RNA. Purified RNA was kept at $-20^{\circ} \mathrm{C}$ before being used for reverse transcription.

Reverse transcription was performed using the TaqMan microRNA Reverse Transcription Kit (Applied Biosystems) according to the instructions by the manufacturer with modifications. The reverse transcription reaction volume was $15 \mu \mathrm{l}$ using $1.5 \mu \mathrm{l}$ Multiscribe reverse transcriptase, $3 \mu \mathrm{l}$ RT-primer-mix (hsamiRNA-126-3p, cel-miRNA-54, and cel-miRNA-238), $1.5 \mu 110 \mathrm{X}$ buffer, $0.3 \mu \mathrm{l} 100 \mathrm{~mm}$ dNTPs, $0.23 \mu \mathrm{l}$ RNAse inhibitor, and $8.47 \mu \mathrm{l}$ RNA purified from plasma. Reverse transcription was performed on an ABI 2720 Thermal Cycler (Applied Biosystems) using a standard protocol $\left(16^{\circ} \mathrm{C}, 30 \mathrm{~min} ; 42^{\circ} \mathrm{C}, 30 \mathrm{~min} ; 85^{\circ} \mathrm{C}, 5 \mathrm{~min}\right.$; hold at $4{ }^{\circ} \mathrm{C}$ ). Reverse-transcribed samples were kept at $-20^{\circ} \mathrm{C}$ until used. 
Reliable and reproducible measurement of circulating miRNA is hampered by the fact that no endogenous miRNA can be used for reliable normalisation, and the amount of total RNA purified from plasma is so small (the concentration of RNA in eluates is typically $\leqslant 5 \mathrm{ng} \mu \mathrm{l}^{-1}$ ) that it is not readily quantified by UV-absorbance measurements. In the absence of absolute determination of miR126 copy number by interpolation to standard curves, which was not possible in this study, we standardised our miRNA amounts based on fixed plasma sample volumes and used spiked-in synthetic non-mammalian miRNA to account for technical variation. This approach enables the comparison of the relative levels of miR-126 between the samples.

cDNA samples and three TaqMan microRNA $(\times 20)$ assays (hsa-miRNA-126-3p, cel-miRNA-54 and cel-miRNA-238; Applied Biosystems) were applied to six 384-well plates according to the manufacturer's instructions. All miR-assays were performed in triplicate (coefficient of variation, 3.6\%). Real-time PCR reaction volume was $10 \mu \mathrm{l}$ for each reaction. Each reaction contained $0.5 \mu \mathrm{l}$ $\times 20$ TaqMan microRNA assay, $5 \mu \mathrm{l} \times 2$ TaqMan Universal PCR Master mix, no AmpErase UNG, $3.5 \mu$ nuclease-free $\mathrm{H}_{2} \mathrm{O}$, and $1 \mu \mathrm{l}$ cDNA. The real-time PCR was performed in the ViiA7 real-time PCR system (Applied Biosystems) using the standard 384-well protocol $\left(50^{\circ} \mathrm{C}, 2 \mathrm{~min} ; 95^{\circ} \mathrm{C}, 10 \mathrm{~min} ; 40\right.$ cycles of $95^{\circ} \mathrm{C}$ for $15 \mathrm{~s}$, and $60{ }^{\circ} \mathrm{C}$ for $60 \mathrm{~s}$; hold at $4{ }^{\circ} \mathrm{C}$ ). Data processing took place using the Applied Biosystems' ViiA7 real-time PCR analysis software (v.1.2.2). This approach provides an estimate of the total amount of stable miRNA-126 in a sample. Thus, it is not possible to determine whether the miRNA originates from microvessicles or miRNA-protein complexes.

In the 384-well plate data analysis the highly reliable auto detectors setting (auto threshold and auto baseline) was chosen for data from all six plates. The average Cq value of hsa- miRNA-126$3 p$ was subtracted from the average Cq values of Cel-miRNA-54 and cel-miRNA-238 for that particular sample yielding the $-\Delta \mathrm{Cq}$ (Cq of cel-miRNAs - Cq of hsa-miRNA-126-3p) values. The $-\Delta \mathrm{Cq}$ values were normalised by the average of $-\Delta \mathrm{Cq}$ values from the whole data set. The normalised $-\Delta \Delta \mathrm{Cq}$ values were used in the further analyses after transformation according to the $2-\Delta \Delta \mathrm{Cq}$ method (Schmittgen and Livak, 2008). The presented estimates for cir-miRNA-126 are thus relative values without a dimension.

Statistics. We report median values with a 95\% confidence interval (CI) and mean values \pm s.d. The comparisons of medians were performed using Wilcoxon rank-sum test and means using an equal-variance $t$-test. Fisher's exact test was used for comparison among categorical parameters. Correlations between continuous variables were quantified using linear regression analyses. Survival functions were estimated by log-rank tests and illustrated by the Kaplan-Meier method. Progression-free survival (PFS) was defined as the time from start of treatment until the first documented tumour progression or death from any cause. The PFS data were censored in 10 cases. Six patients underwent liver resections, two had their primary tumours removed, and two patients underwent radio frequency ablation for liver and lung metastases, respectively. Data were censored from the day of the intervention. All patients were successfully followed until progression. All statistical calculations were carried out using the NCSS statistical software (NCSS Statistical Software, Kaysville, UT, USA, version 2007). $P$ values $<0.05$ were considered significant, and all tests were two-sided.

\section{RESULTS}

Patient characteristics. On the based of the initial screening, 68 patients were available for analyses (Figure 1).
Patient characteristics and baseline cir-miRNA-126 levels are shown in Table 1. A significantly higher baseline cir-miRNA-126 level in the younger half of the patients was demonstrated.

Response to treatment. Three patients stopped treatment early before evaluation and two patients did not have evaluable lesions (too small in size) according to RECIST (Figure 1) and were thus excluded from the response and PFS comparisons.

The mean cir-miRNA-126 level at baseline, first clinical evaluation (after 3 weeks of treatment), and at progression according to treatment response is shown in Figure 2. Four patients had PD (tumour progression of minimum 20\%), $28 \mathrm{SD}$ ( $<30 \%$ tumour regression and $<20 \%$ progression), $29 \mathrm{PR}$ (tumour regression of minimum 30\%), and 2 CR (no visible tumours). There were no significant differences between the mean cir-miRNA-126 levels in the four response groups at baseline and first evaluation. The changes in cir-miRNA-126 during treatment, however, suggested different patterns associated with treatment response. This is illustrated in Figure 3 focusing on the changes from baseline to first clinical evaluation after 3 weeks in nonresponding $(\mathrm{PD}+\mathrm{SD})$ and responding patients $(\mathrm{PR}+\mathrm{CR})$, respectively. Among the non-responding patients, 25 out of the 32 had an increase in cir-miRNA-126 levels compared with 12 out of the 31 responding patients, $P=0.002$. Accordingly, the median change in cir-miRNA-126 levels for the non-responding patients was 0.244 (95\% CI, 0.050-0.565) compared with a median change of -0.374 ( $95 \% \mathrm{CI},-0.472$ to 0.111$)$ in the responding patients, $P=0.002$. Similarly, addressing the changes from baseline to progression, 20 out of the 26 non-responding patients had an increase in cir-miRNA-126 levels compared with 3 out of 21 responding patients, $P<10^{-4}$. Accordingly, the median change in cir-miRNA-126 levels for the non-responding patients was 0.288

\begin{tabular}{|c|c|c|c|}
\hline Characteristic & Number (\%) & $\begin{array}{c}\text { Cir-miRNA-126 } \\
\text { Median }(95 \% \mathrm{CI})\end{array}$ & $P$-value \\
\hline \multicolumn{4}{|l|}{ Gender } \\
\hline Male & $42(62)$ & 1.055 (0.759-1.338) & \multirow[t]{2}{*}{0.80} \\
\hline Female & $26(38)$ & $1.031(0.754-1.416)$ & \\
\hline \multicolumn{4}{|l|}{ Age, years } \\
\hline Median & 66 & & \multirow{4}{*}{0.04} \\
\hline Range & $32-79$ & & \\
\hline$\geqslant 66$ & $35(51)$ & $0.966(0.730-1.047)$ & \\
\hline$<66$ & $33(49)$ & $1.329(0.815-1.435)$ & \\
\hline \multicolumn{4}{|l|}{ ECOG PS } \\
\hline 0 & $46(68)$ & $1.167(0.958-1.369)$ & \multirow[t]{2}{*}{0.09} \\
\hline $1-2$ & $22(32)$ & $0.894(0.583-1.131)$ & \\
\hline \multicolumn{4}{|c|}{ Location of primary tumour } \\
\hline Colon & $37(54)$ & $1.104(0.841-1.416)$ & \multirow[t]{2}{*}{0.13} \\
\hline Rectum & $31(46)$ & 0.967 (0.619-1.338) & \\
\hline \multicolumn{4}{|c|}{ Primary tumour operated } \\
\hline Yes & $22(32)$ & $1.119(0.533-1.406)$ & \multirow[t]{2}{*}{0.60} \\
\hline No & $46(68)$ & $1.022(0.815-1.338)$ & \\
\hline \multicolumn{4}{|c|}{ Metastatic organ sites } \\
\hline 1 & $30(44)$ & $1.001(0.700-1.356)$ & \multirow[t]{2}{*}{0.73} \\
\hline$\geqslant 2$ & $38(56)$ & $1.112(0.759-1.364)$ & \\
\hline \multicolumn{4}{|l|}{$\mathrm{KRAS}^{\mathrm{a}}$} \\
\hline WT & $14(42)$ & $1.143(0.759-1.652)$ & \multirow[t]{2}{*}{0.06} \\
\hline Mut & $19(58)$ & $1.029(0.505-1.200)$ & \\
\hline \multicolumn{4}{|c|}{$\begin{array}{l}\text { Abbreviations: } \quad \mathrm{Cl}=\text { confidence interval; Cir-miRNA-126=circulating microRNA-126; } \\
\text { ECOG PS = Eastern Cooperative Oncology Group Performance Status; KRAS=Kirsten rat } \\
\text { sarcoma viral oncogene homolog; Mut }=\text { mutated; } W T=\text { wild type. Bold values are used to } \\
\text { emphasise the significance of the } P \text {-value }(P<0.05) \text {. } \\
{ }^{\text {a }} \text { Not assessed in all patients. }\end{array}$} \\
\hline
\end{tabular}


(95\% CI, 0.042-0.507) compared with a median change of -0.524

(95\% CI, -0.848 to 0.173 ) in the responding patients, $P=0.0002$.

Further, a positive significant relationship was documented by comparing the changes in tumour size with the corresponding changes in cir-miRNA-126 levels, $r=0.48, P=0.0001$ (Figure 4).

There was no significant relationship between age and treatment efficacy.

Survival analyses. Grouping patients according to the median cirmiRNA-126 level at baseline resulted in a significant separation of the PFS curves (Figure 5A) favouring patients with miRNA-126 levels above the median (median PFS 9.3 months, 95\% CI 8.8-10.6 months) compared with patients with miRNA-126 levels below the median (median PFS 7.3 months (95\% CI 6.7-9.2 months), hazard ratio (HR) 0.53 (95\% CI, 0.29-0.97), $P=0.02$.

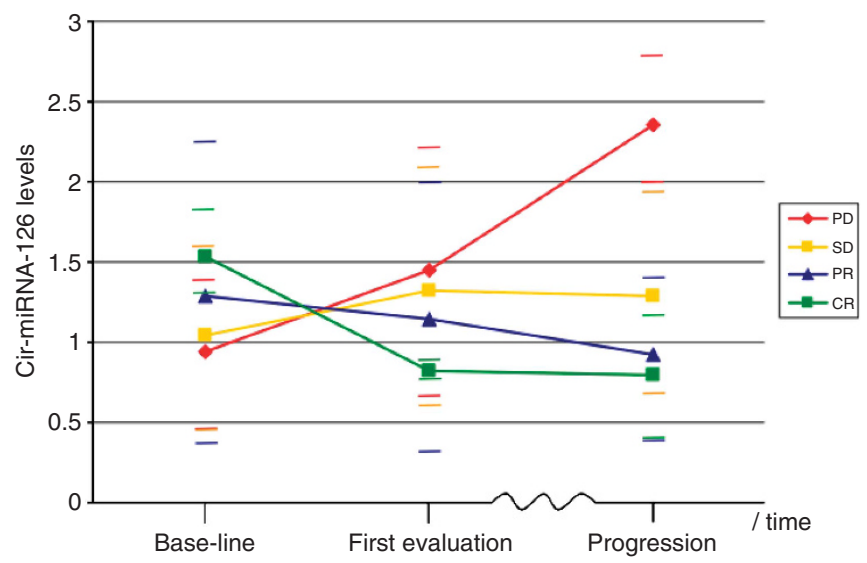

Figure 2. Mean cir-miRNA-126 (cir-miRNA-126) levels according to treatment response (PD, SD, PR, and CR). Horizontal lines mark the respective standard deviation intervals. The broken time line between first clinical evaluation (after 3 weeks) and progression indicates that this time period varies between the patients. As it appears from the overlapping s.d. intervals there were no significant differences between the means of the respective response groups at baseline and first evaluation. The mean cir-miRNA-126 of the PD group was significantly higher than that of the remaining three response groups $(P<0.05)$ at progression. (PD, $N=4),(S D, N=28),(P R, N=29)$, and $(C R=2)$.

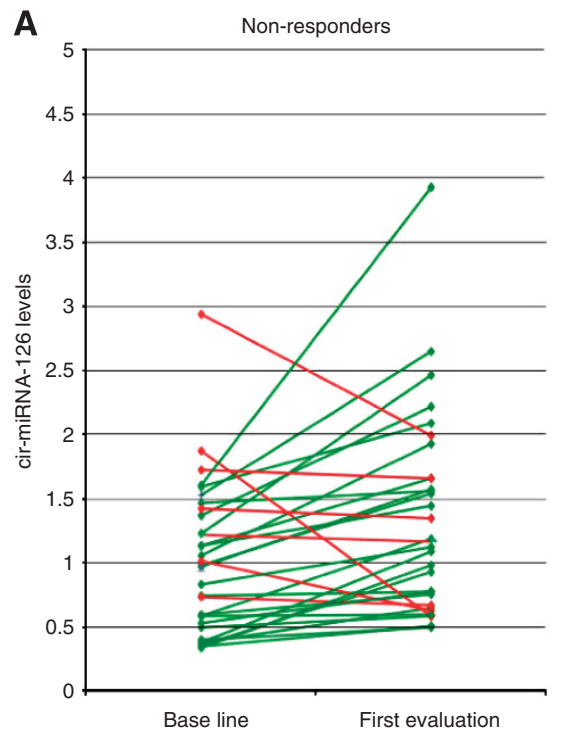

Grouping patients according to the changes in miRNA-126 levels from baseline to first clinical evaluation resulted in a similar, but non-significant separation of the PFS curves (Figure 5B) favouring patients with decreasing miRNA-126 levels (median PFS 9.2 months, 95\% CI 7.3-10.6 months) compared with patients with increasing miRNA-126 levels (median PFS 8.8 months (95\% CI 6.7-9.3 months), HR 0.60 (95\% CI, 0.33-1.09), $P=0.07$.

\section{DISCUSSION}

We analysed cir-miRNA-126 in patients with mCRC treated with first-line chemotherapy combined with bevacizumab and argue for a relationship between tumour response and changes in cirmiRNA-126 levels.

The results documented a relationship between increasing levels of cir-miRNA-126 and a lack of benefit from the treatment, whereas decreasing levels of cir-miRNA-126 were associated with treatment benefit. It is worth noticing that the non-responding patients seem to present with a rather uniform change in cirmiRNA-126 levels, in that 5 of the 7 patients with decreasing levels only had a minor decrease (Figure 3 ). Thus, 30 out of the 32 nonresponding patients exhibited stable or increasing cir-miRNA-126 levels. We are not aware of similar studies in patients with mCRC but Triozzi et al (2012) analysed circulating miRNAs, including cir-miRNA-126, in 21 patients with uveal melanoma treated with adjuvant dacarbazine and interferon- $\alpha-2 b$. They documented changes in cir-miRNA-126 during treatment but no association with outcome. Malka et al (2012) demonstrated an association between high levels of circulating EC and worse RR to first-line chemotherapy + bevacizumab in 99 patients with mCRC. In another setting, Willett et al (2009) demonstrated that patients with rectal cancer treated with neoadjuvant radiotherapy, chemotherapy, and bevacizumab with elevated levels of circulating ECs had a worse outcome. The results from the present study thus agree with the previous reports on the correlation between increasing levels of circulating ECs and vascular damage (Bakouboula et al, 2008). The results from these two studies of patients with CRC may thus reflect a similar mechanism as the one addressed in the present study.

An attempt to explain the possible relationship between the changes in cir-miRNA-126 and tumour response may require

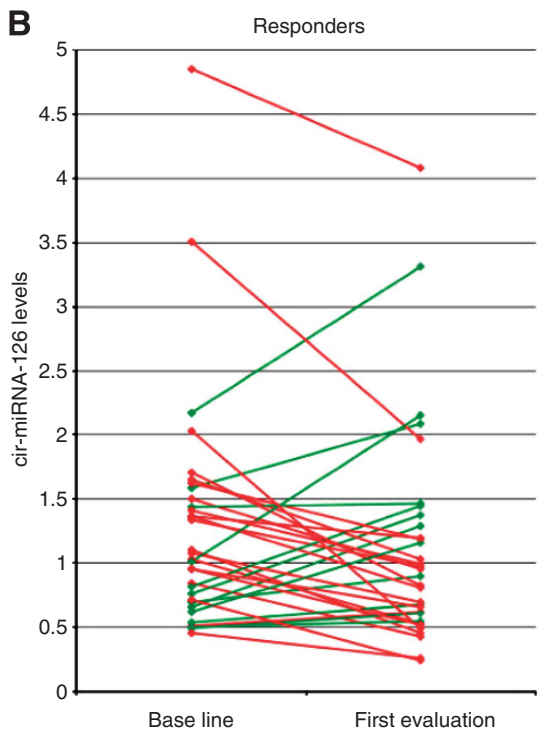

Figure 3. Changes in cir-miRNA-126 (cir-miRNA-126) levels from baseline to first clinical evaluation (after three weeks) in (A) non-responding patients $(N=32)$ and $(B)$ responding patients $(N=31)$. Green and red lines are used to differentiate between the increasing and decreasing miRNA-126 levels, respectively. 
consideration of the normalisation hypothesis by Jain and colleagues (2013). Part of this hypothesis addresses the consequences of too potent/high doses of anti-angiogenic therapy. In such cases, the vessel pruning may become excessive causing necrosis and hypoxia, hampering delivery of drugs such as chemotherapeutics. With the specificity of miRNA-126 for the EC compartment and the established relationship between the increased levels of circulating microvesicles and vascular damage (Bakouboula et al, 2008), the increasing cir-miRNA-126 levels in the present study may reflect an EC-damage associated with bevacizumab treatment. Microvesicles containing miRNA-126 released in response to tissue damage have been shown to induce angiogenesis in recipient cells (Lee et al, 2012), and the increasing levels of cir-miRNA-126 may also reflect bevacizumab resistance leading to renewed stimulation of angiogenesis. Selective release from the tumour-associated blood vessels of the tumour suppressive miRNA-126 (Palma et al, 2012) may constitute a third explanation.

In line with the relationship between cir-miRNA-126 levels and RR, we also detected an association with the actual changes in tumour size during treatment (percent change of predefined target lesions from baseline to first evaluation). This illustration of data primarily served as a visual presentation of the proposed

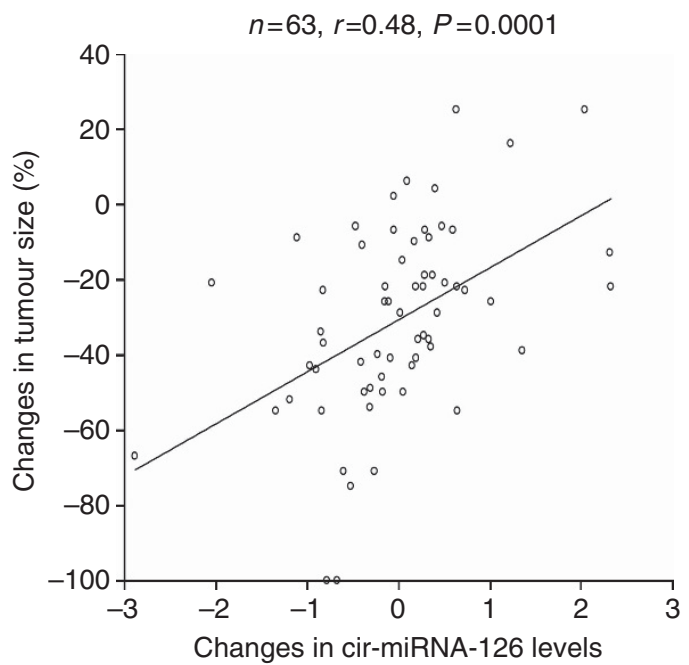

Figure 4. Linear regression plot illustrating the relationship between changes in tumour size according to RECIST (PD: increase of tumour size $>20 \%$; PR: regression of tumour size $>30 \%$; SD: not PD or PR; CR: no visible tumour) and the corresponding changes in circulating miRNA-126 (cir-miRNA-126) levels from baseline. link between treatment efficacy (changes in tumour size) and cirmiRNA-126. Furthermore, it demonstrates that the presented relationship between the two parameters was not just a consequence of the arbitrary cutoffs defined in the RECIST criteria.

Our present results suggest a favourable prognosis in patients with high levels of cir-miRNA-126 at baseline and in patients with decreasing cir-miRNA-126 levels during treatment. These observations are comparable to our previous studies in patients with mCRC based on the analysis of miRNA-126 in tumour tissue (Hansen et al, 2012, 2013). Similar to our results, the relationship between circulating EC and RR in the study by Malka et al (2012) did not translate into a convincing relationship with PFS. In the present study, this may partly be due to a rather high rate of surgical interventions towards the metastatic and primary tumour lesions (15\%), causing these well-responding patients to contribute with limited time for the PFS analyses due to censoring. A recent study of 45 patients with malignant mesothelioma by Tomasetti et al (2012) demonstrated a similar association between low levels of circulating miRNA-126 and a worse prognosis .

So why was baseline cir-miRNA-126 related to PFS and not RR, although changes in cir-miRNA-126 were related to RR and not convincingly to PFS? A possible explanation for this divergence may be provided, if we address cir-miRNA-126 as two independent biomarkers in the two different specific situations (baseline and changes). Baseline cir-miRNA-126 is the result of/influenced by an untreated tumour burden and does not necessarily have anything to do with the treatment intended to be used. The relationship with PFS in this case may thus indicate a pure prognostic value. Changes in cir-miRNA-126, on the other hand, is the consequence of the delivered chemotherapy and bevacizumab and thus reflects an entirely different situation where treatment responsiveness, blood vessel vulnerability and the angiogenic balance in the patient may be the primary mediators of miRNA-126 to the circulation, thus indicating a possible predictive value.

Apart from a possible connection between age and cir-miRNA126 levels there were no significant relationships between patient characteristics and baseline levels to suggest an overall patient and tumour-independent relationship between cir-miRNA-126 and treatment outcome. This is comparable to our previous studies (Hansen et al, 2012, 2013) indicating no relationship between miRNA-126 and patient/tumour characteristics.

In conclusion, the current findings suggest a relationship between early changes in cir-miRNA-126 during first-line chemotherapy + bevacizumab and tumour response in patients with mCRC. This may represent a new predictive biomarker of resistance to anti-angiogenic containing therapies and deserves further research.
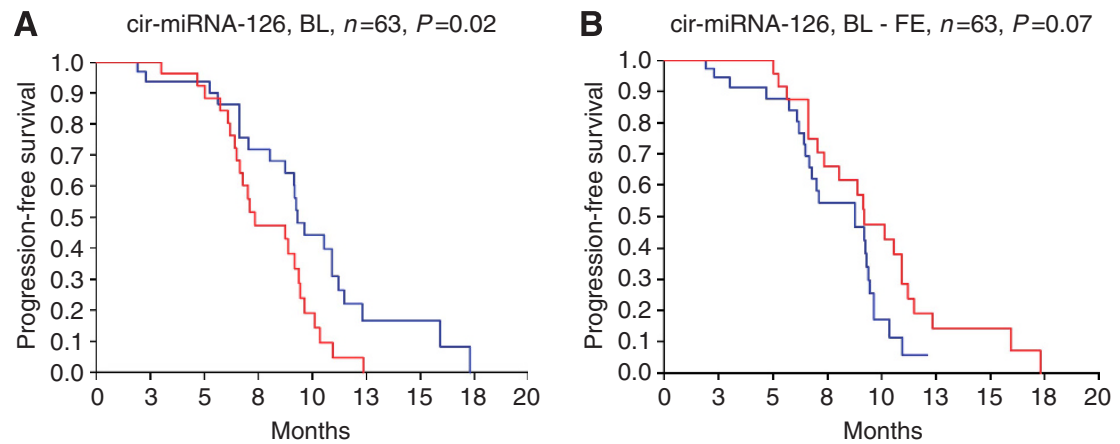

Figure 5. Progression-free survival curves according to circulating miRNA-126 (cir-miRNA-126) levels (A) at baseline (BL) and (B) changes from baseline to first clinical evaluation (BL-FE). The blue curve in (A) represents patients with baseline miRNA-126 levels above the median and the red curve miRNA-126 levels below the median. The red curve in (B) represent patient with decreasing miRNA-126 levels from baseline to first clinical evaluation and the blue curve patients with increasing levels. 


\section{ACKNOWLEDGEMENTS}

We are very thankful for the technical assistance provided by Birgit Roed Sørensen and Lone Frischknecht and for the linguistic editing provided by Karin Larsen. This study was supported by The Cancer Foundation and The Danish Council for Independent Research, none of which had any influence on any part of the study.

\section{CONFLICT OF INTEREST}

The authors declare no conflict of interest.

\section{REFERENCES}

Bakouboula B, Morel O, Faure A, Zobairi F, Jesel L, Trinh A, Zupan M, Canuet M, Grunebaum L, Brunette A, Desprez D, Chabot F, Weitzenblum E, Freyssinet JM, Chaouat A, Toti F (2008) Procoagulant membrane microparticles correlate with the severity of pulmonary arterial hypertension. Am J Respir Crit Care Med 177: 536-543.

Chen X, Ba Y, Ma L, Cai X, Yin Y, Wang K, Guo J, Zhang Y, Chen J, Guo X, Li Q, Li X, Wang W, Zhang Y, Wang J, Jiang X, Xiang Y, Xu C, Zheng P, Zhang J, Li R, Zhang H, Shang X, Gong T, Ning G, Wang J, Zen K, Zhang J, Zhang CY (2008) Characterization of microRNAs in serum: a novel class of biomarkers for diagnosis of cancer and other diseases. Cell Res 18: 997-1006.

Cocucci E, Racchetti G, Meldolesi J (2009) Shedding microvesicles: artefacts no more. Trends Cell Biol 19: 43-51.

Eisenhauer EA, Therasse P, Bogaerts J, Schwartz LH, Sargent D, Ford R, Dancey J, Arbuck S, Gwyther S, Mooney M, Rubinstein L, Shankar L, Dodd L, Kaplan R, Lacombe D, Verweij J (2009) New response evaluation criteria in solid tumours: revised RECIST guideline (version 1.1). Eur J Cancer 45: 228-247.

Esquela-Kerscher A, Slack FJ (2006) Oncomirs - microRNAs with a role in cancer. Nat Rev Cancer 6: 259-269.

Filipowicz W, Bhattacharyya SN, Sonenberg N (2008) Mechanisms of post-transcriptional regulation by microRNAs: are the answers in sight? Nat Rev Genet 9: 102-114.

Ferrara N, Gerber HP, LeCouter J (2003) The biology of VEGF and its receptors. Nat Med 9: 669-676.

Fish JE, Santoro MM, Morton SU, Yu S, Yeh RF, Wythe JD, Ivey KN, Bruneau BG, Stainier DY, Srivastava D (2008) miR-126 regulates angiogenic signaling and vascular integrity. Dev Cell 15: 272-284.

Hansen TF, Christensen Rd, Andersen RF, Sorensen FB, Johnsson A, Jakobsen A (2013) MicroRNA-126 and epidermal growth factor-like domain 7-an angiogenic couple of importance in metastatic colorectal cancer. Results from the Nordic ACT trial. Br J Cancer 109: 1243-1251.

Hansen TF, Soerensen FB, Lindebjerg J, Jakobsen A (2012) The predictive value of microRNA-126 in relation to first line treatment with capecitabine and oxaliplatin in patients with metastatic colorectal cancer. BMC Cancer 8: 83 .

Hunter MP, Ismail N, Zhang X, Aguda BD, Lee EJ, Yu L, Xiao T, Schafer J, Lee ML, Schmittgen TD, Nana-Sinkam SP, Jarjoura D, Marsh CB (2008) Detection of microRNA expression in human peripheral blood microvesicles. PLoS One 3: e3694.

Jain RK (2013) Normalizing tumor microenvironment to treat cancer: bench to bedside to biomarkers. J Clin Oncol 31: 2205-2218.

Kong YW, Ferland-McCollough D, Jackson TJ, Bushell M (2012) microRNAs in cancer management. Lancet Oncol 13: 249-258.

Kosaka N, Iguchi H, Ochiya T (2010) Circulating microRNA in body fluid: a new potential biomarker for cancer diagnosis and prognosis. Cancer Sci 101: 2087-2092.

Lee Y, El AS, Wood MJ (2012) Exosomes and microvesicles: extracellular vesicles for genetic information transfer and gene therapy. Hum Mol Genet 21: 125-134.

Malka D, Boige V, Jacques N, Vimond N, Adenis A, Boucher E, Pierga JY, Conroy T, Chauffert B, Francois E, Guichard P, Galais MP, Cvitkovic F, Ducreux M, Farace F (2012) Clinical value of circulating endothelial cell levels in metastatic colorectal cancer patients treated with first-line chemotherapy and bevacizumab. Ann Oncol 23: 919-927.

McShane LM, Altman DG, Sauerbrei W, Taube SE, Gion M, Clark GM (2005) Reporting recommendations for tumour marker prognostic studies (REMARK). Br J Cancer 93: 387-391.

Mitchell PS, Parkin RK, Kroh EM, Fritz BR, Wyman SK, PogosovaAgadjanyan EL, Peterson A, Noteboom J, O'Briant KC, Allen A, Lin DW, Urban N, Drescher CW, Knudsen BS, Stirewalt DL, Gentleman R, Vessella RL, Nelson PS, Martin DB, Tewari M (2008) Circulating microRNAs as stable blood-based markers for cancer detection. Proc Natl Acad Sci USA 105: 10513-10518.

Mocharla P, Briand S, Giannotti G, Dorries C, Jakob P, Paneni F, Luscher T, Landmesser U (2013) AngiomiR-126 expression and secretion from circulating $\mathrm{CD} 34(+)$ and $\mathrm{CD} 14(+)$ PBMCs: role for proangiogenic effects and alterations in type 2 diabetics. Blood 121: 226-236.

Moore HM, Kelly AB, Jewell SD, McShane LM, Clark DP, Greenspan R, Hayes DF, Hainaut P, Kim P, Mansfield EA, Potapova O, Riegman P, Rubinstein Y, Seijo E, Somiari S, Watson P, Weier HU, Zhu C, Vaught J (2011) Biospecimen reporting for improved study quality (BRISQ). Cancer Cytopathol 119: 92-101.

Ng EK, Chong WW, Jin H, Lam EK, Shin VY, Yu J, Poon TC, Ng SS, Sung JJ (2009) Differential expression of microRNAs in plasma of patients with colorectal cancer: a potential marker for colorectal cancer screening. Gut 58: 1375-1381.

Palma J, Yaddanapudi SC, Pigati L, Havens MA, Jeong S, Weiner GA, Weimer KM, Stern B, Hastings ML, Duelli DM (2012) MicroRNAs are exported from malignant cells in customized particles. Nucleic Acids Res 40: 9125-9138.

Schmittgen TD, Livak KJ (2008) Analyzing real-time PCR data by the comparative C(T) method. Nat Protoc 3: 1101-1108.

Tomasetti M, Staffolani S, Nocchi L, Neuzil J, Strafella E, Manzella N, Mariotti L, Bracci M, Valentino M, Amati M, Santarelli L (2012) Clinical significance of circulating miR-126 quantification in malignant mesothelioma patients. Clin Biochem 45: 575-581.

Triozzi PL, Achberger S, Aldrich W, Singh AD, Grane R, Borden EC (2012) The association of blood angioregulatory microRNA levels with circulating endothelial cells and angiogenic proteins in patients receiving dacarbazine and interferon. J Transl Med 10: 241.

Valadi H, Ekstrom K, Bossios A, Sjostrand M, Lee JJ, Lotvall JO (2007) Exosome-mediated transfer of mRNAs and microRNAs is a novel mechanism of genetic exchange between cells. Nat Cell Biol 9: 654-659.

Wang LG, Gu J (2012) Serum microRNA-29a is a promising novel marker for early detection of colorectal liver metastasis. Cancer Epidemiol 36: 61-67.

Wang S, Aurora AB, Johnson BA, Qi X, McAnally J, Hill JA, Richardson JA, Bassel-Duby R, Olson EN (2008) The endothelial-specific microRNA miR-126 governs vascular integrity and angiogenesis. Dev Cell 15: 261-271.

Weber JA, Baxter DH, Zhang S, Huang DY, Huang KH, Lee MJ, Galas DJ, Wang K (2010) The microRNA spectrum in 12 body fluids. Clin Chem 56: 1733-1741.

Wienholds E, Kloosterman WP, Miska E, varez-Saavedra E, Berezikov E, de BE, Horvitz HR, Kauppinen S, Plasterk RH (2005) MicroRNA expression in zebrafish embryonic development. Science 309: 310-311.

Willett CG, Duda DG, di TE, Boucher Y, Ancukiewicz M, Sahani DV, Lahdenranta J, Chung DC, Fischman AJ, Lauwers GY, Shellito P, Czito BG, Wong TZ, Paulson E, Poleski M, Vujaskovic Z, Bentley R, Chen HX, Clark JW, Jain RK (2009) Efficacy, safety, and biomarkers of neoadjuvant bevacizumab, radiation therapy, and fluorouracil in rectal cancer: a multidisciplinary phase II study. J Clin Oncol 27: 3020-3026.

Zhu N, Zhang D, Xie H, Zhou Z, Chen H, Hu T, Bai Y, Shen Y, Yuan W, Jing Q, Qin Y (2011) Endothelial-specific intron-derived miR-126 is down-regulated in human breast cancer and targets both VEGFA and PIK3R2. Mol Cell Biochem 351: 157-164.

This work is published under the standard license to publish agreement. After 12 months the work will become freely available and the license terms will switch to a Creative Commons AttributionNonCommercial-Share Alike 4.0 Unported License. 